\title{
X-ray emission from wind blown interstellar bubbles
}

\section{ROSAT HRI observations of NGC 6888}

\author{
M. Wrigge and H. J. Wendker
}

Hamburger Sternwarte, Gojenbergsweg 112, 21029 Hamburg, Germany

Received 25 September 2001/ Accepted 27 May 2002

\begin{abstract}
We have observed the stellar wind blown bubble NGC 6888 with the ROSAT HRI. A map and a catalogue of X-ray filaments is derived, the typical filament being a few $0.1 \mathrm{pc}$ in extent with an HRI count rate of a few $10^{-4} \mathrm{~s}^{-1}$. We show that this filamentary structure can qualitatively be modelled as local count rate enhancements due to denser gas near evaporating cool clumps which in turn are seen in the optical and probably are produced by instabilities after the passage of the primary shock front of the expanding bubble.
\end{abstract}

Key words. ISM: bubbles - ISM: individual objects: NGC 6888 - ISM: general - X-rays: ISM

\section{Introduction}

Wind driven interstellar bubbles are an important class of objects for the energy balance of the interstellar medium. Shock fronts are generated by the supersonic expansion of the stellar wind and the analytical models of Weaver et al. (1977) predict the interior of the bubbles to be filled with hot gas. So do hydrodynamic models (i.e. García-Segura \& MacLow 1995a, 1995b). From the observational side we have shown that the prototypical ring nebula NGC 6888 not only is much dimmer at X-rays than predicted but also that the geometry of its $\mathrm{X}$-ray emission does not follow the models (Wrigge et al. 1994, henceforth Paper I). Another bubble, S 308, is marginally consistent with the assumed geometry of the models but the observed flux density implies two orders of magnitude less wind power than is found from direct wind measurements (Wrigge 1999, henceforth Paper II). For NGC 6888 it is excluded via ASCA observations (Wrigge et al. 1998, Paper III of this series) that hotter gas $\left(\geq 10^{7} \mathrm{~K}\right)$ is abundant enough in order to alleviate the problem.

The mapping of NGC 6888 with the ROSAT PSPC (Paper I) showed a second problem namely that the distribution of the X-ray emission could be described as filamentary, matter of fact in Paper I it was estimated that the volume filling factor of the observed gas is about a few percent. As the ROSAT PSPC not really resolved the structures we have obtained ROSAT HRI pointings for NGC 6888. These will be presented and discussed in this paper. In Sects. 2 and 3 we will describe the data reduction and our disentanglement of a number of filaments. In Sect. 4 we will attempt to explain the

Send offprint requests to: H. J. Wendker, e-mail: hjwendker@hs.uni-hamburg.de

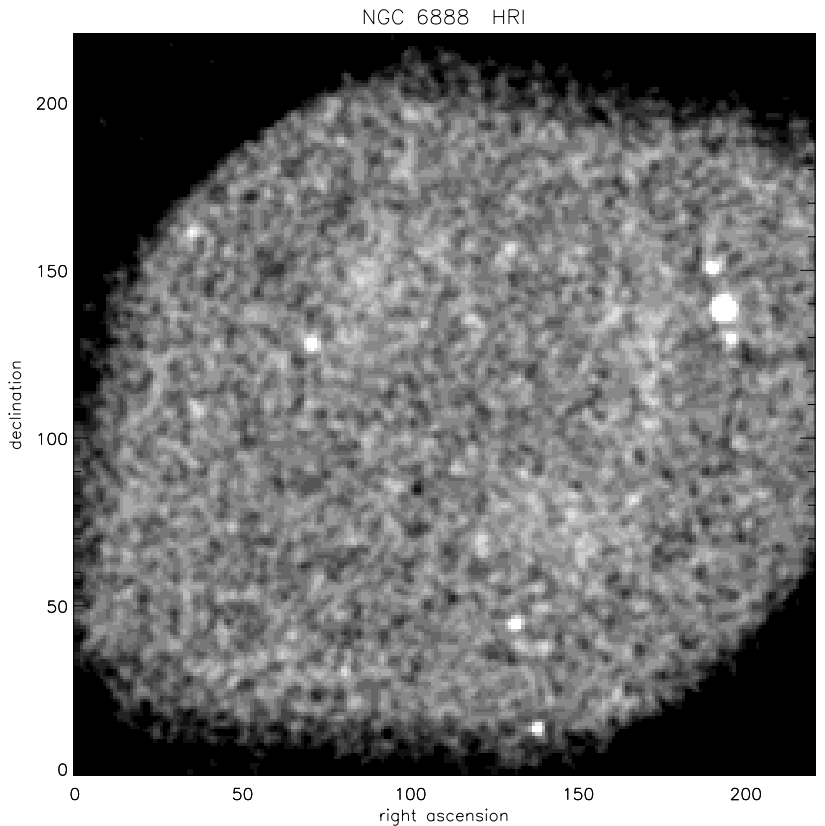

Fig. 1. Grayscale image of the merged HRI obervations of NGC 6888 with a logarithmic scaling for an 8 " pixel size (white corresponding to 17.7 photons per pixel or more, black to 7.1 photons per pixel or less). The axes are in pixel number with $8^{\prime \prime}$ steps; $(0,0)$ corresponds to $\left(20^{\mathrm{h}} 14^{\mathrm{m}} 07.83^{\mathrm{s}}, 38^{\circ} 05^{\prime} 27^{\prime \prime}\right)$. A few point sources and the outline of 3 emission complexes can be recognized. In general they cover the northern, western and southern complexes of optical filaments like the overlay of the PSPC data on an $\mathrm{H} \alpha$ photo as given in Fig. 2 of Paper I.

behaviour of the filaments of NGC 6888 as cool clumps $\left(10^{4} \mathrm{~K}\right)$ which are evaporated by the expanding hot gas $\left(>10^{6} \mathrm{~K}\right)$ which was generated by the high speed stellar wind. 


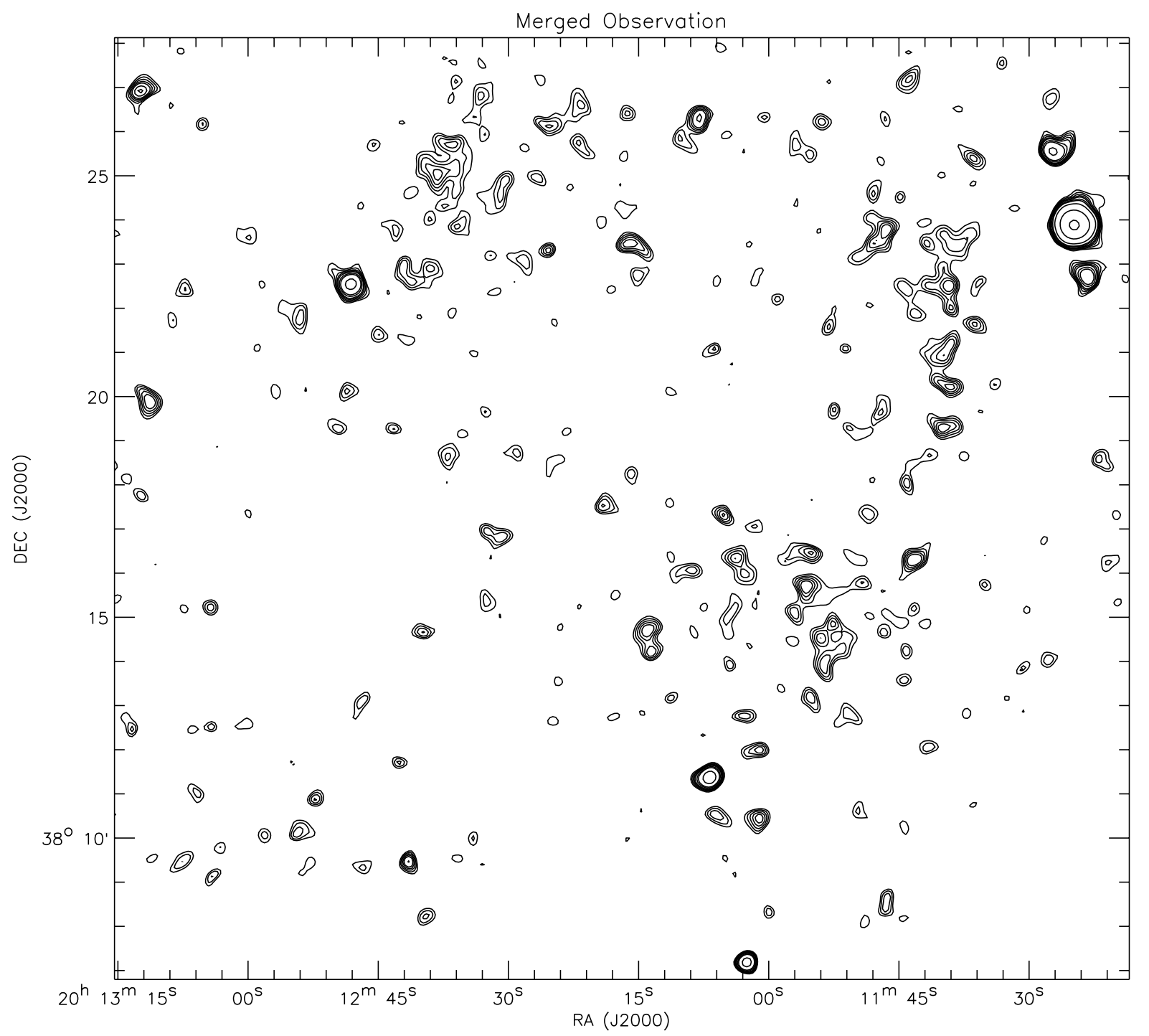

Fig. 2. Contour map of the merged pointings of NGC 6888. The first contour is $2 \sigma_{\mathrm{pp}}$ above the background of $5.2 \times 10^{-3} \mathrm{~s}^{-1} \operatorname{arcmin}^{-2}(\mathrm{see} t \mathrm{text})$. The step size is $0.5 \sigma_{\mathrm{pp}}=0.2 \times 10^{-3} \mathrm{~s}^{-1} \mathrm{arcmin}^{-2}$ for the first 5 contours. The following contours are then $5,10,30$ and $100 \sigma_{\mathrm{pp}}$. (Source \#1 of Table A1 is outside the map area in the southern pointing only.)

\section{ROSAT HRI observations}

The ROSAT PSPC map of NGC 6888 (see Figs. 2 and 3 of Paper I) with an effective angular resolution of 50" shows that the X-ray emission is brightest in the northern and southern parts of the nebula. From the general description of ROSAT and its instrumentation (Trümper 1983; Pfeffermann et al. 1986) it follows that the field of view of the HRI (about $38^{\prime}$ diameter) covers NGC 6888 (long axis about $18^{\prime}$ ) but that the interesting parts would be in areas with already deteriorating angular resolution (from $4^{\prime \prime}$ to $>20^{\prime \prime}$ ). In the planning stage we expected the filaments to have a width comparable or smaller than the best possible HRI angular resolution. In order to obtain an indication of the extend of a large number of them the observations were split into two pointings centered on the northern and southern maxima. The data were taken between April 1994 and April 1995 with total exposure times of about 55 and $67 \mathrm{ks}$, respectively. Both pointings overlap considerably.
The raw data immediately indicated that almost all filaments were resolved contrary to our expectation. The signal-to-noise ratio in surface brightness is therefore quite low at the highest possible resolution $\left(4^{\prime \prime}\right)$. In order to get physical information the pointings had to be smoothed and merged which was done in the following way with standard routines (for details of reduction procedures see Zimmermann et al. 1994). The field of view corrections (vignetting etc.) were applied first to the individual fields. Then the individual pointings were smoothed. After a few trials the combination of a pixel size of 4 " and a Gaussian smoothing with a FWHM of $20^{\prime \prime}$ appeared to be the optimum (note that this leaves a small gradient in resolution from centre to edge). These two individual maps were then added. In the worst case (field edges) we add maps with effective angular resolutions of $22^{\prime \prime}$ and $29^{\prime \prime}$. This final map has an effective exposure time of $122 \mathrm{ks}$. A grayscale version of this merged image is given in Fig. 1. The background level was found from a patch in the southeastern part of the merged 
map (about $87 \operatorname{arcmin}^{2}$ ) which seemed free of any real structure to be $5.2 \times 10^{-3} \mathrm{~s}^{-1} \operatorname{arcmin}^{-2}$. A few smaller patches in other parts of the map gave similar results and we conclude that the background in the merged map is uniform. (The number is equivalent to having an average number of approximately 2.8 photons per 4 "'-pixel after smoothing.) The pixel-to-pixel variation turned out to be $\sigma_{\mathrm{pp}}=0.4 \times 10^{-3} \mathrm{~s}^{-1} \operatorname{arcmin}^{-2}$ (or $7 \%$ ). Contours encompassing an area the size of the resolution element or larger were then taken to represent real features if they are $2 \sigma_{\mathrm{pp}}$ above the background level. A map with such properties is shown in Fig. 2. A comparison with the PSPC map in Paper I shows an overall agreement but many differences in detail. PSPC structures mainly fall apart into several filaments but no really new features are seen. We will comment upon these differences further below.

\section{The filaments}

The observations were undertaken in order to see whether the X-ray emission of NGC 6888 could be described as a set of filaments. As a first step we produced a catalogue of such features in the following way. Each local maximum surrounded by at least two contours is called a filament (threshold value $>3 \sigma$ ). The lowest contour was approximated by an ellipse and the long and short axis of the ellipse are used as a measue of the extend of the filament. The number of photons were summed inside the lowest contour and the background of $5.2 \times 10^{-3} \mathrm{~s}^{-1}$ arcmin $^{-2}$ properly scaled to the total area subtracted. This is taken as the number of photons of the filament. Note that this is not a volume fitting procedure but in principle a lower limit through restricting the area to be inside the lower contour. The source area was determined by counting the number of pixels inside the lowest contour.

In order not to contaminate the filament list with point sources we tested each feature which looked small and had roundish contours. It was considered a point source when the fit of a two-dimensional Gaussian did not reveal an extension beyond the resolution limit (see above) and did not result in a significant ellipticity. In this way we found one candidate filament to be a real point source (source \#11 in Table A1), and one case of a blend of a filament and a point source (source \#12 and filament \#f22). One object considered a point source in the PSPC map of Paper I (source \#4) turned out to be resolved and hence is regarded a filament. During this search we also found one filament quite outside the optical outline of the nebula. The list of the HRI point sources in the field is given in the Appendix and briefly discussed there. During this procedure a few candidates due to noise spikes were discarded.

In this way 36 filaments were found, about a factor of 2 more than expected from the PSPC map when simply counting the number of local maxima in Paper I. They are listed in Table 1. Column 1 lists a simple running number of the objects and Cols. 2 and 3 their J2000 coordinates estimated from a map. Using a distance of $1.8 \mathrm{kpc}$ (as in Paper I) one can convert the axis lengths of the ellipses into linear extends of the filaments. The smallest number $(0.21 \mathrm{pc})$ corresponds to the resolution. These values are given in Cols. 4 and 5. Column 6 contains the total number of observed photons. The quoted uncertainty is not a standard error but mainly contains the possible uncertainty due to the separation procedure (weak extended signal on top of a noisy background). Assuming an oblate geometry one can calculated a filament volume $V$ as given in Col. 7. The number of photons from Col. 6 is converted to a luminosity in the HRI band as follows. Paper I shows that the PSPC observations can be described by a RaymondSmith-plasma (Raymond \& Smith 1977) with a typical temperature of $2 \times 10^{6} \mathrm{~K}$. Taking the RS spectrum, the detector characteristic (Zimmermann et al. 1994) and an absorbing ISM with an HI column density of $3 \times 10^{21} \mathrm{~cm}^{-2}$ (Paper I) we obtained an energy conversion factor of $E C F=2 \times 10^{9} \mathrm{erg}^{-1} \mathrm{~cm}^{2}$. The resulting luminosities $L_{\mathrm{X}}$ are given in Col. 8. Taking an average cooling factor of $\Lambda\left(2 \times 10^{6} \mathrm{~K}\right) \sim 2.6 \times 10^{-23} \mathrm{ergs} \mathrm{s}^{-1} \mathrm{~cm}^{3}$ in the ROSAT band for the above mentioned temperature (uncertain by about a factor of 2) one can also calculate the typical volume density of the hot gas via $n=\sqrt{L_{X} /(V \cdot \Lambda)}($ Col. 9).

If we add up all the photons in the filaments in Table 1 a total count rate for NGC 6888 of $\approx 0.014 \mathrm{~s}^{-1}$ results. This is about $1 / 7$ th of the PSPC count rate derived in Paper I $\left(\approx 0.11 \mathrm{~s}^{-1}\right)$. Considering that the sensitivity of the HRI is about $1 / 3.5$ of that of the PSPC we conclude that the HRI observations have missed about half the available photons. This must be due to our smoothing procedure combined with the $2 \sigma$ criterium for defining a filament. These "surplus" photons must be distributed as a diffuse component between the filaments. This will also qualitatively account for the differences between the appearance of NGC 6888 in the PSPC and in the HRI which was mentioned in the beginning.

We conclude that about half the total X-ray emission from NGC 6888 consists of small filaments. A typical X-ray filament is a few tenth of a pc long, its short extension is most often not resolved and therefore less than $0.2 \mathrm{pc}$. Its luminosity in the ROSAT HRI band is several $10^{31} \mathrm{erg} \mathrm{s}^{-1}$. The volume density of this gas is a few $\mathrm{cm}^{-3}$.

\section{Evaporating clumps}

The X-ray morphology of wind blown bubbles in analytical models is governed by the effect of heat conduction on the large scales (see the classical paper by Weaver et al. 1977). For NGC 6888 we have shown in Paper I that the low temperature emission does not originate in matter with large-scale distribution but the high temperature emission may do so (Paper III). Here we want to show that the filaments we found in the HRI observations may originate in small scale density fluctuations (called cool clumps) which evaporate within the expanding already shocked (hot) gas. We will treat the problem as far as possible by analytical means.

Heat conduction leads to an evaporation of the cool clump which in turn results in special temperature and density profiles in this volume. For the spherically symmetric, stationary, and time independent case an analytical solution was derived by Cowie \& McKee (1997) for the temperature profile $T(r)$ and the mass loss rate $\dot{M}_{\text {clump }}$ under classical heat conduction (diffusive transport, unsaturated case) to

$T(r)=T_{\mathrm{f}} \cdot\left(1-R_{\text {clump }} / r\right)^{2 / 5}$ 
Table 1. ROSAT HRI filaments in NGC 6888, observed and derived parameters.

\begin{tabular}{|c|c|c|c|c|c|c|c|c|c|}
\hline$(1)$ & (2) & (3) & (4) & $(5)$ & (6) & (7) & $(8)$ & $(9)$ & $(10)$ \\
\hline $\begin{array}{c}\text { N6888 } \\
\#\end{array}$ & $\begin{array}{c}\alpha \\
\mathrm{J} 2000 \\
20^{\mathrm{h}}+ \\
\end{array}$ & $\begin{array}{c}\delta \\
\mathrm{J} 2000 \\
38^{\circ}+\end{array}$ & $\begin{array}{c}\text { Major } \\
\text { axis } \\
{[\mathrm{pc}]}\end{array}$ & $\begin{array}{l}\text { Minor } \\
\text { axis } \\
{[\mathrm{pc}]}\end{array}$ & $\begin{array}{c}\text { Total } \\
\text { number } \\
\text { of counts }\end{array}$ & $\begin{array}{c}V \\
{\left[10^{53} \mathrm{~cm}^{3}\right]}\end{array}$ & $\begin{array}{c}L_{\mathrm{x}} \\
{\left[10^{31} \mathrm{erg} \mathrm{s}^{-1}\right]}\end{array}$ & $\begin{array}{c}\mathrm{n} \\
{\left[\mathrm{cm}^{-3}\right]}\end{array}$ & Remarks \\
\hline f01 & $11^{\mathrm{m}} 36^{\mathrm{s}}$ & $21.3^{\prime}$ & 0.28 & 0.21 & $20 \pm 13$ & 2.5 & 3.2 & 2.2 & \\
\hline f02 & 1136 & 25.4 & 0.31 & 0.21 & $25 \pm 15$ & 3.1 & 4.0 & 2.2 & \\
\hline f03 & 1139 & 22.5 & 0.64 & 0.28 & $87 \pm 27$ & 17.5 & 12.8 & 1.7 & \\
\hline f04 & 1139 & 23.6 & 0.56 & 0.42 & $86 \pm 28$ & 20.3 & 13.6 & 1.6 & \\
\hline f05 & 1140 & 19.3 & 0.36 & 0.25 & $50 \pm 20$ & 5.0 & 7.9 & 2.5 & \\
\hline f06 & 1140 & 20.2 & 0.42 & 0.21 & $41 \pm 18$ & 5.6 & 6.5 & 2.1 & \\
\hline f07 & 1141 & 21.1 & 0.50 & 0.25 & $67 \pm 24$ & 9.6 & 10.6 & 2.1 & \\
\hline f08 & 1143 & 16.3 & 0.54 & 0.28 & $53 \pm 21$ & 12.6 & 8.4 & 1.6 & \\
\hline f09 & 1144 & 27.2 & 0.33 & 0.23 & $33 \pm 17$ & 3.9 & 5.2 & 2.3 & \\
\hline $\mathrm{f} 10$ & 1148 & 23.6 & 0.69 & 0.28 & $102 \pm 30$ & 20.5 & 16.1 & 1.7 & \\
\hline f11 & 1153 & 14.4 & 0.81 & 0.43 & $145 \pm 36$ & 43.3 & 23.0 & 1.4 & blend \\
\hline $\mathrm{f} 12$ & 1154 & 15.6 & 1.04 & 0.33 & $125 \pm 34$ & 54.7 & 19.8 & 1.2 & blend (of 3?) \\
\hline $\mathrm{f} 13$ & 1155 & 13.2 & 0.31 & 0.21 & $24 \pm 15$ & 3.1 & 3.8 & 2.2 & \\
\hline f14 & 1156 & 16.5 & 0.50 & 0.22 & $51 \pm 22$ & 8.4 & 8.1 & 1.9 & \\
\hline f15 & 1201 & 10.4 & 0.31 & 0.25 & $39 \pm 18$ & 3.7 & 6.2 & 2.5 & \\
\hline f16 & 1202 & 12.0 & 0.32 & 0.21 & $29 \pm 16$ & 3.3 & 4.6 & 2.3 & \\
\hline $\mathrm{f} 17$ & 1203 & 12.8 & 0.28 & 0.21 & $19 \pm 13$ & 2.5 & 3.0 & 2.1 & \\
\hline $\mathrm{f} 18$ & 1204 & 16.2 & 0.47 & 0.27 & $62 \pm 24$ & 9.2 & 9.8 & 2.0 & \\
\hline f19 & 1205 & 17.3 & 0.27 & 0.21 & $25 \pm 15$ & 2.4 & 4.0 & 2.5 & \\
\hline $\mathrm{f} 20$ & 1206 & 10.5 & 0.34 & 0.21 & $27 \pm 16$ & 3.7 & 4.3 & 2.1 & \\
\hline $\mathrm{f} 21$ & 1209 & 16.1 & 0.38 & 0.21 & $29 \pm 17$ & 4.7 & 4.6 & 1.9 & \\
\hline $\mathrm{f} 22$ & 1210 & 25.1 & 0.57 & 0.21 & $25 \pm 15$ & 10.5 & 4.0 & 0.7 & blend with source \#12 \\
\hline $\mathrm{f} 23$ & 1214 & 14.5 & 0.53 & 0.31 & $81 \pm 26$ & 13.4 & 12.8 & 1.9 & \\
\hline f24 & 1215 & 23.4 & 0.44 & 0.22 & $55 \pm 22$ & 6.5 & 8.7 & 2.3 & \\
\hline $\mathrm{f} 25$ & 1219 & 17.5 & 0.31 & 0.21 & $40 \pm 10$ & 3.7 & 6.4 & 2.6 & \\
\hline $\mathrm{f} 26$ & 1222 & 26.5 & 0.32 & 0.21 & $35 \pm 19$ & 3.3 & 5.5 & 2.6 & \\
\hline $\mathrm{f} 27$ & 1223 & 25.8 & 0.31 & 0.21 & $23 \pm 15$ & 3.1 & 3.6 & 2.1 & \\
\hline $\mathrm{f} 28$ & 1225 & 26.2 & 0.35 & 0.21 & $33 \pm 18$ & 3.9 & 5.2 & 2.3 & \\
\hline f29 & 1231 & 26.6 & 0.54 & 0.21 & $56 \pm 24$ & 9.4 & 8.9 & 1.9 & \\
\hline $\mathrm{f} 30$ & 1232 & 16.8 & 0.42 & 0.21 & $38 \pm 10$ & 6.8 & 6.0 & 1.8 & \\
\hline $\mathrm{f} 31$ & 1235 & 26.6 & 0.47 & 0.22 & $35 \pm 19$ & 7.5 & 5.6 & 1.8 & \\
\hline $\mathrm{f} 32$ & 1238 & 18.7 & 0.31 & 0.21 & $32 \pm 10$ & 3.7 & 5.1 & 2.3 & \\
\hline $\mathrm{f} 33$ & 1238 & 25.1 & 0.85 & 0.55 & $195 \pm 42$ & 60.9 & 30.9 & 1.4 & blend \\
\hline f34 & 1240 & 22.9 & 0.57 & 0.31 & $70 \pm 26$ & 15.4 & 11.1 & 1.7 & blend \\
\hline $\mathrm{f} 35$ & 1255 & 21.9 & 0.42 & 0.28 & $65 \pm 25$ & 7.6 & 10.3 & 2.3 & \\
\hline $\mathrm{f} 36$ & 1310 & 19.9 & 0.35 & 0.24 & $92 \pm 25$ & 4.5 & 14.6 & 3.5 & outside optical image \\
\hline
\end{tabular}

Table 2. Parameters of a few characteristic models when the bubble temperature $T_{\mathrm{f}}$ and density $n_{\mathrm{f}}$ are given. For further description see text.

\begin{tabular}{cccccccccc}
\hline \hline $\begin{array}{c}\text { Model } \\
\text { number }\end{array}$ & $\begin{array}{c}T_{\mathrm{f}} \\
{[\mathrm{K}]}\end{array}$ & $\begin{array}{c}n_{\mathrm{f}} \\
{\left[\mathrm{cm}^{-3}\right]}\end{array}$ & $\begin{array}{c}t_{\mathrm{ev}} \\
{[\text { years }]}\end{array}$ & $\sigma_{0}$ & $\begin{array}{c}\mathrm{cts}_{\infty} \\
{\left[\mathrm{s}^{-1}\right]}\end{array}$ & $\begin{array}{c}\mathrm{cts}_{\mathrm{cl}}\left[\mathrm{s}^{-1}\right] \\
\text { unsaturated }\end{array}$ & $\begin{array}{c}\mathrm{cts}_{\mathrm{cl}}\left[\mathrm{s}^{-1}\right] \\
\text { saturated }\end{array}$ & $\begin{array}{c}\Delta_{\mathrm{cl}}\left[\mathrm{s}^{-1}\right] \\
\text { unsaturated }\end{array}$ & $\begin{array}{c}\Delta_{\mathrm{cl}}\left[\mathrm{s}^{-1}\right] \\
\text { saturated }\end{array}$ \\
\hline 1 & $10^{7}$ & 0.07 & $188 \cdot \mu \cdot n$ & 295.8 & $1.25 \times 10^{-4}$ & $1.49 \times 10^{-4}$ & $1.26 \times 10^{-4}$ & $0.25 \times 10^{-4}$ & $0.01 \times 10^{-4}$ \\
2 & $5 \times 10^{6}$ & 0.14 & $222 \cdot \mu \cdot n$ & 38.2 & $3.25 \times 10^{-4}$ & $3.43 \times 10^{-4}$ & $3.32 \times 10^{-4}$ & $0.18 \times 10^{-4}$ & $0.07 \times 10^{-4}$ \\
3 & $2 \times 10^{6}$ & 0.35 & $245 \cdot \mu \cdot n$ & 2.0 & $2.33 \times 10^{-4}$ & $2.01 \times 10^{-4}$ & $2.24 \times 10^{-4}$ & $-0.32 \times 10^{-4}$ & $-0.09 \times 10^{-4}$ \\
4 & $10^{6}$ & 0.7 & $790 \cdot \mu \cdot n$ & 0.33 & $3.14 \times 10^{-5}$ & $2.48 \times 10^{-5}$ & $2.70 \times 10^{-5}$ & $-0.64 \times 10^{-5}$ & $-0.44 \times 10^{-5}$ \\
\hline
\end{tabular}

and

$\dot{M}_{\text {clump }}=8.25 \times 10^{5} \cdot T_{\mathrm{f}}^{5 / 2} \cdot\left(R_{\text {clump }} / 1 \mathrm{pc}\right) / \ln \Lambda$

$T_{\mathrm{f}}$ and $n_{\mathrm{f}}$ are the temperature and density of the medium at large distances from the clump. $R_{\text {clump }}$ is the clump radius and the Coulomb logarithm $\ln \Lambda \approx 29.7+\ln \left(n^{-0.5} T / 10^{6}\right)$.
For the density profile the additional assumption of about constant pressure (subsonic streaming) has to be made (Cowie \& McKee 1997; Böhringer \& Hartquist 1987). Then

$n(r)=n_{\mathrm{f}} \cdot\left(T_{\mathrm{f}} / T(r)\right)$.

Introducing the so called saturation parameter $\sigma_{\mathrm{o}}$ Dalton \& Balbus (1993) found a solution for both cases, saturated 
(i.e. large mean free path length for electrons) and unsaturated heat conduction where

$\sigma_{\mathrm{o}}=\left(2 \cdot \kappa_{\mathrm{f}} \cdot T_{\mathrm{f}}\right) /\left(25 \cdot \rho_{\mathrm{f}} \cdot c_{\mathrm{f}}^{3} \cdot R_{\text {clump }}\right)$

for the unsaturated case and

$\sigma_{\mathrm{o}}=\left(1.1735 \times 10^{6} \sqrt{\mu} \cdot T_{\mathrm{f}}^{2}\right) /\left(n_{\mathrm{f}} \cdot R_{\text {clump }} \ln \Lambda\right)$

for the saturated case. Saturation sets in for $\sigma_{\mathrm{o}}>1$. The formal density profile remains as above.

The free parameters are $T_{\mathrm{f}}$ and $\rho_{\mathrm{f}}$ given the radius of the evaporating clump $R_{\text {clump. As }}$ we are only interested in the emission at X-rays which starts to become relevant for $T_{\mathrm{f}}>5 \times 10^{5} \mathrm{~K}$ the underlying assumption that the temperature inside the clump is close to zero instead of the realistic $\sim 10^{4} \mathrm{~K}$ (photoionization by the WR-star) should not be critical. Missing spectral information the HRI observations only restrict one of the two parameters $T_{\mathrm{f}}$ and $\rho_{\mathrm{f}}$. In the classical analytical theory (Weaver et al. 1977) a further constraint is possible by assuming pressure equilibrium between the interior of the bubble and the clump. If we take from Paper I that this quantity is around $P=7 \times 10^{5} \mathrm{~K} \mathrm{~cm}^{-3}$ this fixes the product of $T_{\mathrm{f}}$ and $\rho_{\mathrm{f}}$ and allows us to calculate the excess of X-ray photons at the clump position for a given temperature. The linear scaling then needs the clump radius. For this we assume that the clumps observed by Mitra (1990) in [OIII] are the ones which we observe in their evaporating phase. Mitra (1990) obtained a typical clump radius of $6^{\prime \prime}$ or $1.7 \times 10^{17} \mathrm{~cm}$. In this way we have calculated four different sets called models 1 to 4 in Table 2 . The $T_{\mathrm{f}}$ and corresponding $n_{\mathrm{f}}$ values cover a range of temperatures of the surrounding gas from $10^{7} \mathrm{~K}$ (the upper limit from ASCA) to $10^{6} \mathrm{~K}$ (the lower limit from the PSPC). From the deduced parameters the evaporation time $t_{\mathrm{ev}}=M_{\text {clump }} / \dot{M}_{\text {clump }}$ depends on the mean molecular weight $\mu$ and the clump density $\mathrm{n}$ and representative numbers are given in Col. 4. Furtheron, the saturation parameter $\sigma_{\mathrm{o}}$, the HRI surface brightness without the presence of a clump $\mathrm{cts}_{\infty}$, the HRI surface brightnesses with a clump $\mathrm{cts}_{\mathrm{cl}}$ for the unsaturated and saturated cases, and the resulting surface brightness differences $\Delta_{\mathrm{cl}}$ for both cases are given. The latter ones are taken from the curves $T(r)$ (Fig. 3), $\rho(r)$ (Fig. 4) and cts(r) (Fig. 5). Figure 5 shows that one single filament contributes but a small amount of excess photons. It is in principle not due to an enhanced temperature but due to the local enhancement of the density just after the gas leaves the clump surface.

\section{Discussion}

Table 2 and Figs. 3-5 indicate that the visibility of a clump in the HRI data strongly depends on the temperature of the hot gas in the bubble interior. At low $T_{\mathrm{f}}$ the clump emission folded with the HRI sensitivity even produces a smaller X-ray flux than would be observed without a clump (models 3 and 4). Only when the interior gas becomes hot enough (models 1 and 2) an observable excess is achieved. Thus indeed the gas should have a temperature beyond a few $10^{6} \mathrm{~K}$ before we could observe the filaments as we do. At these temperatures saturation plays a key role for the visibility which on the other hand produces less photon excesses than the unsaturated case (Fig. 5).

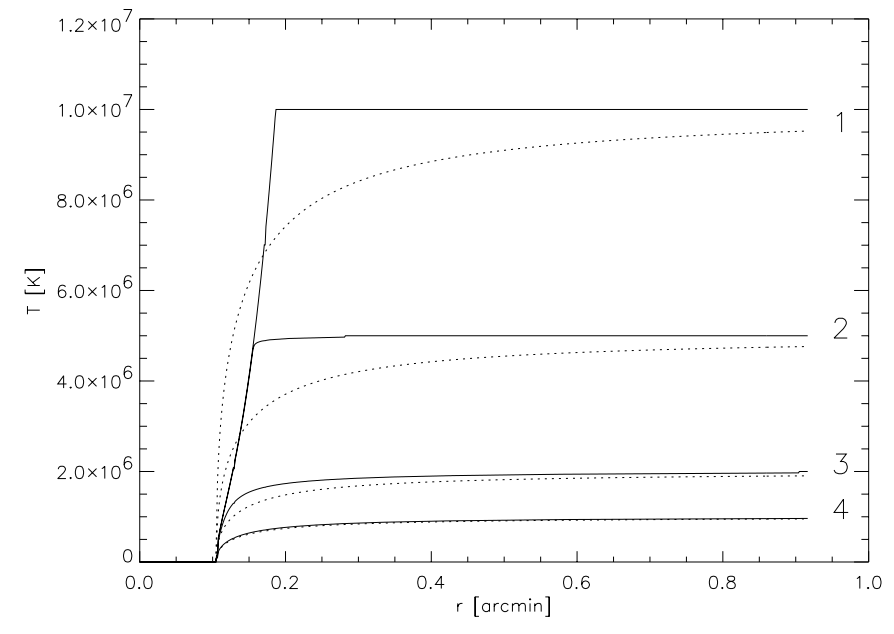

Fig. 3. Temperature profile of an evaporating clump. The angular scale corresponds to a clump radius of $1.7 \times 10^{17} \mathrm{~cm}$ at the bubble distance of $1.8 \mathrm{kpc}$. Dashed curves result from unsaturated heat conduction and solid curves from saturated one. The numbers refer to the models in Table 2.

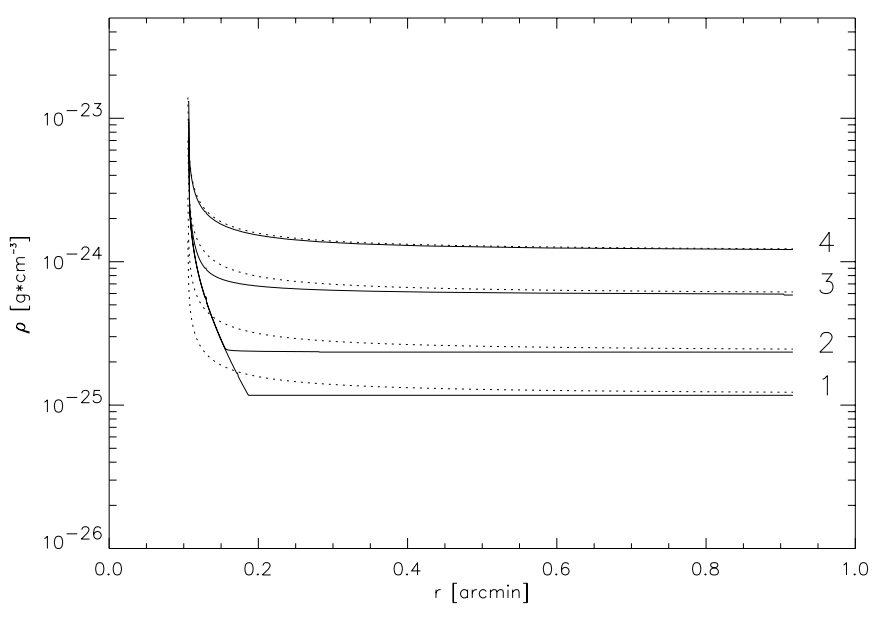

Fig. 4. Same as Fig. 3, but for the density profile.

In Sect. 3 we showed that a typical filament has count rate of $3 \times 10^{-4} \mathrm{~s}^{-1}$ and in its unresolved case an extent of $<0.2 \mathrm{pc}$. To match the observations one needs in the saturated case a few hundred clumps with the properties derived by Mitra (1990) and in the unsaturated case a few tens. Given the total volume this numbers do not present geometric difficulties. Naturally we have in mind that the clumps themselves are generated by Rayleigh-Taylor type instabilities in the primary expanding shock front of the bubble.

Our explanation of the filamentary X-ray structure in NGC 6888 is supported on the observational side by the general filamentary appearance at other wavelengths (i.e. Wendker et al. 1975 at optical and radio, Dickel \& Wendker 1977; Parker 1978 in $\mathrm{H} \alpha$ ). It is, however, impossible to correlate optical and $\mathrm{X}$-ray filaments because of the vastly different reolutions. Even inside our smallest angular scale of around $20^{\prime \prime}$ usually several optical filaments are to be found the resolution there typically around $1 "$. This becomes even more difficult when looking at the HST images presented by Moore et al. (2000) which 
Table A.1. ROSAT HRI point sources in and around NGC 6888.

\begin{tabular}{|c|c|c|c|c|c|}
\hline (1) & (2) & (3) & (4) & (5) & (6) \\
\hline $\begin{array}{c}\text { NGC } 6888 \\
\#\end{array}$ & $\begin{array}{l}\text { ROSAT } \\
\text { designation }\end{array}$ & $\begin{array}{c}\alpha \\
\mathrm{J} 2000 \\
20^{\mathrm{h}}+ \\
\end{array}$ & $\begin{array}{c}\delta \\
\mathrm{J} 2000 \\
38^{\circ}+ \\
\end{array}$ & $\begin{array}{c}\text { count } \\
\text { rate } \\
10^{-3} \mathrm{~s}^{-1}\end{array}$ & $\begin{array}{l}\text { idenfication } \\
\text { or } \\
\text { remarks }\end{array}$ \\
\hline 01 & RXJ2011.0+3806 & $11^{\mathrm{m}} 02.9^{\mathrm{s}}$ & $06^{\prime} 22^{\prime \prime}$ & 4.2 & very faint reddish star \\
\hline 02 & RXJ2011.4+3824 & 1124.8 & 2357 & 12.4 & HD 192020 \\
\hline 03 & RXJ2011.4+3825 & 1127.0 & 2538 & 1.4 & empty \\
\hline 04 & RXJ2011.7+3827 & - & - & - & removed, filament \\
\hline 05 & RXJ2012.1+3821a & 1206.9 & 2105 & 0.25 & HD 193162, WR 136 \\
\hline 06 & RXJ2012.1+3821b & - & - & $<0.15$ & at PSPC position \\
\hline 07 & RXJ2012.8+3822 & 1248.5 & 2236 & 1.3 & empty \\
\hline 08 & RXJ2013.2+3826 & 1312.7 & 2700 & 0.7 & HDE 228376, Ту3151-2805 \\
\hline 09 & RXJ2011.3+3822 & 1123.2 & 2248 & 0.8 & faint star at northern edge \\
\hline 10 & RXJ2012.0+3807 & 1202.7 & 0714 & 1.0 & empty \\
\hline 11 & RXJ2012.1+3811 & 1207.1 & 1126 & 1.1 & empty \\
\hline 12 & RXJ2012.1+3826 & 1208.3 & 2621 & 0.45 & blend with \#f22; faint star at southeast edge \\
\hline 13 & RXJ2012.6+3809 & 1241.4 & 0929 & 0.3 & empty \\
\hline 14 & XTE J2012+381 & - & - & $<0.15$ & at optical position (Hynes et al. 1999) \\
\hline
\end{tabular}

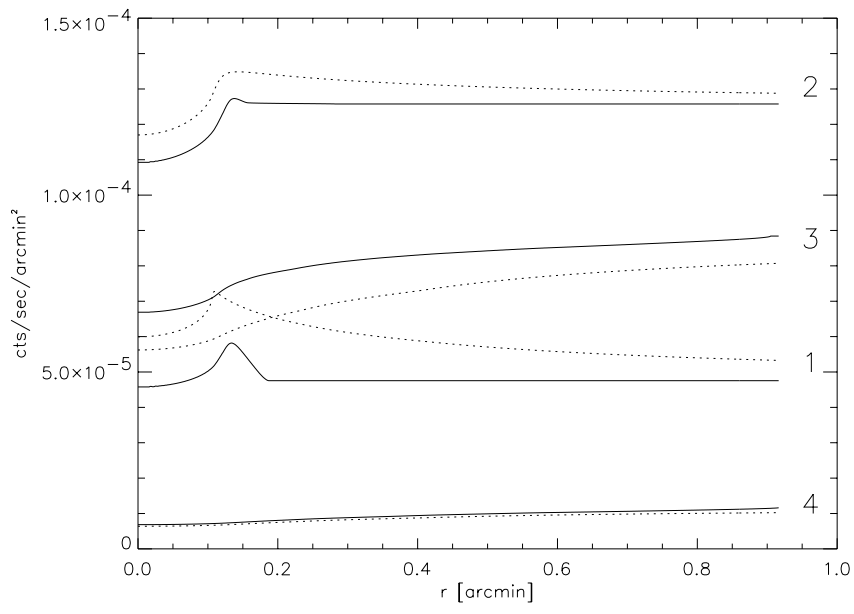

Fig. 5. Same as Fig. 3, but for the surface brightness profile.

show bewildering complicated small scale structures on scales around $0.1^{\prime \prime}$ called clumps by them. These may be the brightest individual features we are looking for. Only one of our filaments, \#f31, covers the small HST image size, but its centre is situated in the left half of the image where there is a very bright spot of many blended filaments the yellowish colour of which indicating the existence of all three kinds of clumps discussed by Moore et al. (2000).

On the theoretical side Pittard et al. (2001) looked at the effects of mass-loading a stellar wind with material from evaporating clumps which is what we suggest happens in our X-ray filaments. Pittard et al. (2001) conclude that depending on the amount of mass-loading the resulting bubble can be hotter in the interior than closer to the edge which would be compatible with our deductions here and in Paper III.

It remains not understood how the temperatur and density distribution in the bubble interior as a whole is produced considering that the summed up observed X-ray emission is still far less than the avalaible energy in the wind luminosity although Pittard et al. (2001) suggest that the right amount of mass-loading could alleviate the problem. This unsolved topic occurs in all 4 papers of this series. A hint that interactions of the wind quite beyond the outline of the optically visible nebula occurs can be seen in the existence of the filaments f 35 and f 36 although we can not rule out with absolute certainty that these structures are a superposition of closeby point sources conspiring to mimick a filament. Lying in the minor axis direction $\mathrm{f} 36$ is in projection about 1.5 times further away from the WR star than the furthest filaments along the major axis.

Acknowledgements. We would like to thank the MPE and its ROSAT team for making these observations possible. We acknowledge many fruitful discussions with Y.-H. Chu, G. García-Segura, M.-M. MacLow, U. Wessolowski, and L. Wisotzki. MW is greatly indebted to the Institut für Astronomie und Astrophysik of the University of Kiel for hospitality during this work. Part of this work was supported by the BMBF (DARA FKZ 50 OR 9308 and DLR FKZ 50 OR 9604).

\section{Appendix A: Point sources}

\section{A.1. Listing and short discussion}

Here we summarize our results for the point sources in tabular form (Table A.1). The numbering and designation scheme for the first 8 sources is taken from Paper I. Source \#9 was not resolved from source \#2 in the PSPC data. Additional sources are then added. The positions are from the Gaussian fits to the HRI data unless otherwise noted. The numerical errors are a few seconds of arc only but could be as large as $15^{\prime \prime}$. The average difference to the PSPC positions from Paper I is less than $20^{\prime \prime}$. The count rates were obtained from an eye estimated optimal extraction circle and an immediately surrounding background count ring of equal size. The uncertainty is about $15 \%$ for the fainter sources dropping to less than $5 \%$ for the strongest. Taking the sensitivity of the HRI to be $1 / 3.5$ of that of the PSPC the HRI count rates average to about $80 \%$ of those of the PSPC. Considering that the most likely counterparts are stellar objects and that those are mostly variable the agreement seems satisfactory. The last 
column of Table A.1 contains some remarks and the result of a quick identification attempt on the ESO online digitized sky survey with a $15^{\prime \prime}$ error circle around each position. Only one new useful identification was found, namely HDE 228376 for source \#8. The Tycho catalogue (ESA 1997) gives a colour index of $(B-V) \sim 0.46^{\mathrm{m}}$ and the HDE classification is A0. Nothing else seems to be known.

\section{A.2. Remarks on XTE J2012+381}

A transient X-ray source, designated XTE J2012+381, was discovered in May 1998 by Remmillard et al. (1998) and later found to be a so called soft X-ray transient with counterparts from the optical to the radio wavelengths range. For a referenced listing of these detections see i.e. Hynes et al. (1999). While presenting the HRI observations we point out that this source position is covered by $\mathrm{X}$-ray images obtained by various satellites.

XTE J2012+381 is most likely identical with the hitherto unidentified source \#4 in the EINSTEIN field from April 1979 on a count rate level around $0.007 \mathrm{~s}^{-1}$ as found by Bochkarev (1988). This source was not seen with EXOSAT in Dec. 1983 and Jun. 1984 (Kähler et al. 1987), with the ROSAT PSPC in April 1991 (Paper I), and with the ROSAT HRI in April 1994 and $1995.3 \sigma$ count rate upper limits are all between 0.002 and 0.001 .

It is tempting to interpret the time span between the 1979 source detection and the new findings in Mai 1998 as a rough indication of a long "duty cycle" (18 years and 10 months). Although small integer fractions of this number certainly can not be ruled out especially as the typical length of these transients is not known, only $1 / 6$ and $1 / 8$ come close to the ROSAT HRI and PSPC observations, respectively.

\section{References}

Bochkarev, N. G. 1988, Nature, 332, 518

Böhringer, H., \& Hartquist, T. W. 1987, MNRAS, 228, 915

Cowie, L. L., \& McKee, C. F. 1977, ApJ, 211, 135

Dalton, W. W., \& Balbus, S. A. 1993, ApJ, 404, 625

Dickel, H. R., \& Wendker, H. J. 1977, A\&A, 29, 269

ESA 1997, The Hipparcos and Tycho Catalogues, ESA SP-1200

García - Segura, G., \& MacLow, M.-M. 1995a, ApJ, 455, 145

García - Segura, G., \& MacLow, M.-M. 1995b, ApJ, 455, 160

Hynes, R. L., Roche, P., Charles, P. A., \& Coe, M. J. 1999, MNRAS, 305, L49

Kähler, H., Ule, T., \& Wendker, H. J. 1987, Ap\&SS, 135, 105

Mitra, P. 1990, Ph.D. Thesis, Rice University

Moore, B. D., Hester, J. J., \& Scowen, P. A. 2000, AJ, 119, 2991

Parker, R. A. R. 1978, ApJ, 224, 873

Pfeffermann, E., Briel, U. G., Hippmann, H., et al. 1986, Proc. SPIE, 733,519

Pittard, J. M., Dyson, J. E., \& Hartquist, T. W. 2001, A\&A, 367, 1000

Raymond, J. C., \& Smith, B. W. 1977, ApJS, 35, 419

Remmillard, R., Levine, A., \& Wood, A. 1998, IAU Circ. 6620

Trümper, J. 1983, Adv. Sp. Res., 2, 241

Weaver, R., McCray, R., Castor, J., Shapiro, P., \& Moore, R. 1977, ApJ, 218, 377

Wendker, H. J., Smith, L. F., Israel, F. P., Habing, H. J., \& Dickel, H. R. 1975, A\&A, 42, 173

Wrigge, M. 1999, A\&A, 343, 599 (Paper II)

Wrigge, M., Chu, Y.-H., Magnier, E. A., \& Kamata, Y. 1998, Lecture Notes in Phys., ed. D. Breitschwert, M. J. Freyberg, \& J. Trümper, 506, 425, (Paper III)

Wrigge, M., Wendker, H. J., \& Wisotzki, L. 1994, A\&A, 286, 219 (Paper I)

Zimmermann, H. U., Becker, W., Belloni, T., et al. 1994, EXSAS User's Guide, MPE Report 257, Garching 